\title{
Subcarrier Slip Detection for High-Order BOC signals
}

\author{
Moisés Navarro-Gallardo ${ }^{(1,2)}$, Gustavo López-Risueño ${ }^{(2)}$, Jose Antonio García-Molina ${ }^{(2)}$, \\ Massimo Crisci $^{(2)}$ and Gonzalo Seco-Granados ${ }^{(1)}$ \\ (1) Universitat Autònoma de Barcelona (UAB), Spain \\ (2) European Space Agency (ESA), The Netherlands \\ e-mails: \{moises.navarro.gallardo;gustavo.lopez.risueno;jose.antonio.garcia.molina;massimo.crisci\}@esa.int \\ \{gonzalo.seco\}@uab.cat
}

\begin{abstract}
The most recent generation of Global Navigation Satellite Systems (GNSS) are implementing Binary Offset Carrier (BOC) modulation. The advantage of BOC signals is that the main lobe of the correlation is very narrow, but on the other hand, they present side peaks, i.e. multiple stable tracking points. For high-order BOC signals, the amplitude of the side peaks can be similar to the amplitude of the main one or even exceed it under specific scenarios. Some techniques to mitigate the code ambiguity exploit the fact that BOC signals can be understood as the sum of two BPSK signals. Therefore, BOC signals are composed by three components: the code, the subcarrier and the carrier. Code gives unambiguous but noisy measures, whereas that the subcarrier tracking gives ambiguous but precise measurements. The technique presented in this paper solves the ambiguity combining these two delays: the same principle as the carrier smoothing techniques is exploited. Additional, a new subcarrier slip detector is presented in order to detect the subcarrier slips and reset the code smoothing. This paper is focused on highorder BOC signals as the BOC $(15,2.5)$ present in the European Galileo System. The performance of the new algorithm has been evaluated though several simulations using the DLR channel model and other synthetic scenarios.
\end{abstract}

\section{INTRODUCTION}

The BOC (Binary Offset Carrier) signals have been chosen for the new generation of GNSS (Global Navigation Satellite System) as Galileo, the upgrade of the GPS system, the future Chinese (COMPASS/BEIDOU) and Indian (GAGAN) systems. These signals were expected to provide not only better precision in the estimation of the delay, but also more robustness to multipath effects. These contributions are combined with the direct signal causing an error in the estimation of the position.

The BOC ambiguity issue is a well-known problem: mistaking a side peak of the auto-correlation function with the main peak and hence producing an error in the computation of the position. This issue becomes more challenging as the order of the BOC increases. For instance, the auto-correlation of BOC $(15,2.5)$ signal has the side peak only at 9.7 meters of the main one, and its value is around 0.89 (normalized assuming that the maximum is equal to one), for $\operatorname{BOC}(10,5)$ the closer side lobe is at 14.65 meters and its value is around 0.74 . Several methods to mitigate the ambiguity have been presented in the literature. One of the first ones is the Bump Jumping algorithm [1]. It uses two extra correlators placed in the nearest peaks to the tracked one. If the power of one of the two extra correlators is bigger than the tracked one, all the correlators jump towards that one. A true kind of unambiguous techniques are the so-called BPSK-like, that are based on seeing the BOC signals as the sum of two BPSK signals [2] [3]. They can be filtered by one filter that has enough bandwidth for both BPSK, or each BPSK can be filtered though different filters centred at each frequency lobe. In [4] the authors analyse the behaviour of using only one BPSK signal instead of the combination of two. Another unambiguous technique that achieves the BPSK, or close, shape is based on taking out the sub-carrier component as it is usually done with the carrier [5]. Although these methods are able to fully mitigate the ambiguity, they lose all the multipath mitigation properties of the BOC signals. The Double Estimator (DE) [6] is based on the use of three loops: Phase Lock Loop (PLL) for the phase delay, Delay Lock Loop (DLL) for the code delay and a new one for the subcarrier delay, the Sub-carrier Lock Loop (SLL). It assumes that, the code and subcarrier delays could be different. Therefore, a 2D correlation is obtained, combining both dimensions, i.e. the DLL and SLL. The DLL dimension has the same shape than the BPSK-like. Furthermore, The idea of the DE technique is to correct the SLL using the DLL. If the different between them is bigger than half subcarrier cycle, the SLL measurement is moved towards the DLL by an integer of the subcarrier. In [7] the author presents different ways to combine the two measures obtained with the DE technique.

In [8] a new idea in order to mitigate the ambiguity problem is presented. It is based on the code and the subcarrier measurements. The goal is to smooth the code: the same idea as in carrier smoothing but using two code measurements instead of one phase and one code measurements. Using the BPSK-like techniques an unambiguous but noisy delay is achieved. The delay estimated using the full BOC signal (subcarrier delay) is ambiguous but less noisy. There is a big similitude between these two delays and the ones that are used in carrier smoothing strategy: the code measurements are unambiguous but noisy, whereas that the carrier measurements are less accurate but ambiguous. One of the carrier smoothing problems is that the carrier and the code are delayed differently by the ionosphere. Hence, a bias between both measurement is introduced and it is time variant. This effect limits the smoothing time. Since the code and sub-carrier components are close to the same frequency, the ionosphere effect is 
practically the same for both code delays.

As the carrier phase, the subcarrier component could suffer subcarrier slips. Therefore, the smoothing must be reset when a subcarrier slip takes place. This paper presents a new algorithm in order to detect the subcarrier slips that is insensitive to possible distortions in the cross-correlation due to the filtering or channel/model effects. This technique can be seen as an improvement of [8].

The remaining of this paper is organized as follows. In Section II the signal model and the fact that the BOC signals can be expressed as the sum of BPSK is presented. The Section III exposes the mathematical expressions of the smoothing strategy and its common version carrier smoothing. The new subcarrier slip detector algorithm and the complete codesubcarrier smoothing with slip detector is presented in Section IV. The results and the conclusions are presented in Section $\mathrm{V}$ and Section VI.

\section{Signal Model}

BOC signals can be expressed as

$$
s(t)=\sum_{k} c_{k} p_{s c}\left(t-k T_{c}\right)
$$

where is the amp $c_{k}(t)$ PRN code sequence waveform, $T_{c}$ is the chip period and $p_{s c}$ is the subcarrier waveform: $\operatorname{sign}\left[\cos \left(2 \pi f_{s c} t\right)\right]$ for $\mathrm{BOCcos}$ and $\operatorname{sign}\left[\sin \left(2 \pi f_{s c} t\right)\right]$ for BOCsin. Nevertheless, the fact that the BOC signals can be tracked as the sum of two BPSK is a well-known ambiguity mitigation method. Actually, it can be shown that BOCcos pulses can be written as sum of infinite sinusoids using the Fourier series as

$$
p_{s c}(t)=\sum_{n=1}^{\infty} \frac{4(-1)^{(n+1)}}{\pi(2 n-1)} \cos \left(w_{0}(2 n-1) t\right) p(t),
$$

where $w_{o}=2 \pi / T_{o}, T_{o}$ is the inverse of the sub-carrier frequency, and $p(t)$ is a square pulse of duration equal to inverse the chip rate. The amplitude is decreasing as $n$ increases. Therefore, one may track BOC signals as two independent BPSK centred at $+/$ - the subcarrier frequency. Several algorithms, take the advantage of the code component such as the Double Estimator [6], the Subcarrier Phase Cancellation [5] amount others. Although all the techniques are quite different, the aim is exactly the same: achieving an unambiguous delay exploiting the code component of the BOC signals.

\section{SMOOTHING STRATEGY}

\section{A. Hatch Filter}

The most common smoothing technique is based on the Hatch filter [9], [10]. The idea is to smooth an unbiased but noisy measurement using a biased but non-noisy measurement. For instance, the code is smoothed using the carrier measurements. Fig. 1 shows the block diagram of the smoothing technique. The Hatch filter can be expressed as

$$
\bar{X}[k]=\frac{1}{\alpha} X[k]+\left(1-\frac{1}{\alpha}\right) \bar{X}[k-1],
$$

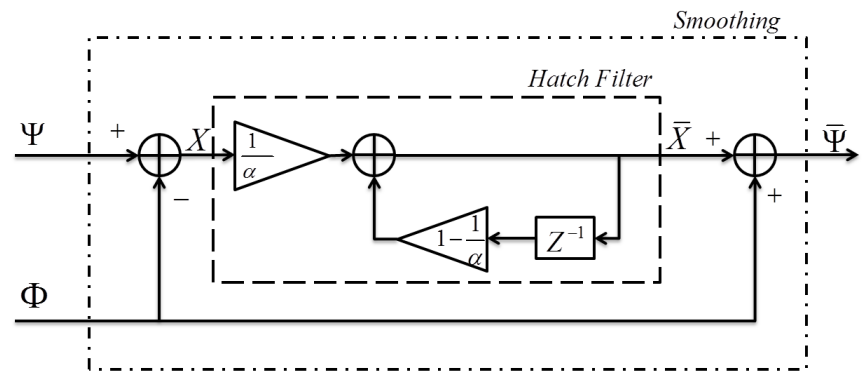

Fig. 1. Smoothing block diagram

where $\alpha$ is the smoothing time constant in terms of number of samples, $\bar{X}[k]$ is the filtered version of $X=\Psi-\Phi$, i.e. the difference between the two estimated measurements, which can be defined as

$$
\begin{aligned}
& \Psi=r+I_{\Psi}+w_{\Psi}, \\
& \Phi=r+I_{\Phi}+w_{\Phi}+\lambda,
\end{aligned}
$$

where $r$ is the distance between the satellite and the receiver and it is the same for both measurements, $I_{\Psi}$ and $I_{\Phi}$ are the ionosphere delays for both measurements and could be different, $w_{\Psi}$ and $w_{\Phi}$ are the thermal noise and multipath effect and $\lambda$ is the ambiguity term. The smoothed measurement can be written as

$$
\bar{\Psi}[k]=\bar{X}[k]+\Phi[k] .
$$

Substituting (4) and (3) in (5), the smoothed measurement can be written as

$$
\begin{aligned}
& \bar{\Psi}[k]=\left(r[k]+I_{\Phi}[k]+w_{\Phi}[k]+\lambda\right)+ \\
& +\frac{1}{\alpha}\left(r[k]+I_{\Psi}[k]+w_{\Psi}[k]\right) \\
& +\frac{1}{\alpha}\left(-r[k]-I_{\Phi}[k]-w_{\Phi}[k]-\lambda\right)+ \\
& +\left(1-\frac{1}{\alpha}\right)\left(\bar{r}[t-1]+\bar{I}_{\Psi}[t-1]+\bar{w}_{\Psi}[t-1]\right)+ \\
& +\left(1-\frac{1}{\alpha}\right)\left(-\bar{r}[t-1]-\bar{I}_{\Phi}[t-1]-\bar{w}_{\Phi}[t-1]-\lambda\right),
\end{aligned}
$$

where we have used the following definitions

$$
\begin{aligned}
& \bar{I}_{\Psi}[k]=\frac{1}{\alpha} I_{\Psi}[k]+\left(1-\frac{1}{\alpha}\right) \bar{I}_{\Psi}[t-1], \\
& \bar{I}_{\Phi}[k]=\frac{1}{\alpha} I_{\Phi}[k]+\left(1-\frac{1}{\alpha}\right) \bar{I}_{\Phi}[t-1], \\
& \bar{w}_{\Psi}[k]=\frac{1}{\alpha} w_{\Psi}[k]+\left(1-\frac{1}{\alpha}\right) \bar{w}_{\Psi}[t-1], \\
& \bar{w}_{\Phi}[k]=\frac{1}{\alpha} w_{\Phi}[k]+\left(1-\frac{1}{\alpha}\right) \bar{w}_{\Phi}[t-1] .
\end{aligned}
$$

The expression in (6) can be written as

$$
\begin{aligned}
& \bar{\Psi}[k]=r[k]+ \\
& +I_{\Phi}[k]+w_{\Phi}[k]+\bar{I}_{\Psi}[k]-\bar{I}_{\Phi}[k]+\bar{w}_{\Psi}[k]-\bar{w}_{\Phi}[k] .
\end{aligned}
$$

The last two terms are the filtered noise of both measurements. Compare to the sampled noise, these two terms can be neglected. $\bar{I}_{\Psi}[k]$ and $\bar{I}_{\Phi}[k]$ are the filtered or smoothed delays due to the ionosphere.

\section{B. Carrier Smoothing}

The carrier smoothing method is well known in the GNSS community: the carrier measurements are used jointly with the code measurements in order to reduce the code noise. In this case, the generalized variables presented in the previous section can be formulated as $\Psi=\rho$ (code measurements )and 
$\Phi=\phi$ (phase measurements). The delay introduced by the ionosphere is well documented [11] $I_{\Psi}=-I_{\Phi}=I$. Then, (8) can be written as

$$
\bar{\rho}[k]=r[k]+I[k]+w_{\phi}[k]+2 \bar{I}[k]+\bar{w}_{\rho}[k]-\bar{w}_{\phi}[k] .
$$

The last equation shows the dependence on the previous evolution ionosphere effect. As it is time depending a large integration time can cause an unacceptable bias.

\section{Code smoothing strategy}

In [8] a new smoothing strategy is presented, its schematic is shown in Fig. 2. There are three independent loops: The full BOC and the two BPSK tracking loops. Nevertheless, the phase measurements $\left(\theta_{\text {carrier }}\right)$ from the full BOC can be supplied to the BPSK loops. The code measurements from the two BPSK loops $\left(\tau_{U}\right.$ and $\left.\tau_{L}\right)$ are combined to each other getting the $\tau_{\text {BPSK }}$ measurement. Finally, It is smoothed with the full BOC measurement $\left(\tau_{\mathrm{BOC}}\right)$ obtaining the unambiguous and smoothed $\bar{\tau}$ measurement.

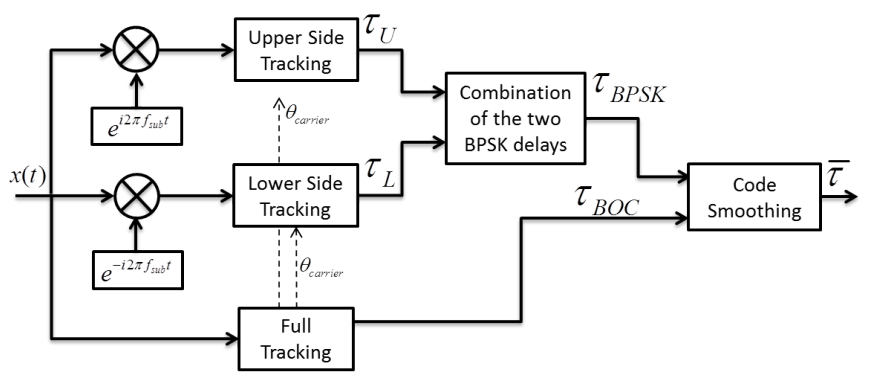

Fig. 2. Code-Subcarrier Smoothing schematic

The generalized variables can be reformulated for code smoothing as $\Psi=\tau_{\text {BPSK }}$ and $\Phi=\tau_{\text {BOC }}$. Assuming that both delays are affected in the same way by the ionosphere, i.e. $I_{\Psi}=I_{\Phi}=I$, (8) is written as

$$
\begin{aligned}
& \bar{\tau}[k]= \\
& =r[k]+I[k]+w_{\tau_{\mathrm{BOC}}}[k]+\bar{w}_{\tau_{\mathrm{BPSK}}}[k]-\bar{w}_{\tau_{\mathrm{BOC}}}[k] .
\end{aligned}
$$

The result achieved is very interesting. The measurement does not depend on the past evolution of the ionosphere effect. Therefore a large smoothing time can be done. It has to be taken into account that a change in the ambiguity produces the same effect as a cycle slip. Moreover, if a large smoothing time is set the filtered terms in the equation can be neglected. Then, the measurement can be approximated as

$$
\bar{\tau}[k] \approx r[k]+I[k]+w_{\tau_{\mathrm{BOC}}}[k] .
$$

In order to reduce the time of convergence, when code smoothing starts, $\alpha$ increases linearly until it reaches its defined value.

This algorithm solves the ambiguity problem. However, if during the tracking a subcarrier slip takes place, i.e. the tracking point goes from one peak of the ACF to another one, the convergence time could reach huge values, depending on the smoothing time constant. Therefore, an algorithm in order to detect the subcarrier slips is needed. It is presented in the following section.

\section{SUbCARRIER SLIP DETECTOR}

The concept of cycle slip is well known for the GNSS carrier phase measurements. Therefore, jumps of integer numbers of wavelengths can be detected. Depending on the number of frequencies and the number of satellites, different methods are used for cycle-slip detection and correction. In [12] and [13] several cycle slips detectors can be found.

In this paper the same concept of cycle slips is exploited but for the subcarrier component. Instead of detect jumps of $20 \mathrm{~cm}$ (the wavelength of the L1 carrier), jumps of 10 meters have to be detected. Mainly, one may think in multi-frequency detectors or single-frequency detectors. The goal of the first ones is to get rid of the geometry without introducing the noise and multipath code errors. For instance, combining the E1BC and E5a signals of Galileo. The single-frequency detectors combine the code and phase in order to get rid of the geometry.

Since BOC modulations have the subcarrier and the carrier component, the same concepts of the multi-frequency detectors can be applied. Hence, the phase could be used in order to detect subcarrier slips. Nevertheless, this method has been discarded, because the high probability of cycle slip.

In this paper a new method for subcarrier slip detection is presented. It is based on the combination between the code and the subcarrier measurements in order to get rid of the geometry. Hence, the input of the detector is

$$
d[k]=\tau_{B O C}[k]-\tau_{B P S K}[k]
$$

where $\tau_{B O C}$ is the subcarrier measurement and $\tau_{B P S K}$ is the code measurement. This combination has some similarities with respect to the code minus carrier combination. However, there is no ionospheric impact, since the delay introduced by the ionosphere is the same for both delays [8].

The code measurements could be really affected by the noise and multipath, reaching up few subcarrier cycles. Therefore, the combination must be smoothed. In this paper the Algorithm 1: Subcarrier Slip Detector, that takes into account the mean and variance of the combination, has been implemented in order to detect subcarrier slips.

The detection is based on the computation of the mean and the standard deviation of the input combination $(d)$. A subcarrier slip is detected when the actual input combination differs from the estimated mean over $K$ times the standard deviation. The initial standard deviation can be initialised to $S_{d}[0]=\lambda / 2$, i.e. half the subcarrier period. When a subcarrier slip is detected, the algorithm is reset.

Fig. 3 shows the new receiver structure. The subcarrier slip detector has been added to the previous code-subcarrier smoothing structure. When the detector declares a subcarrier slip, the code smoothing algorithm is reset. It is noteworthy that the algorithm can be implemented for any order and any kind of BOC modulation. 

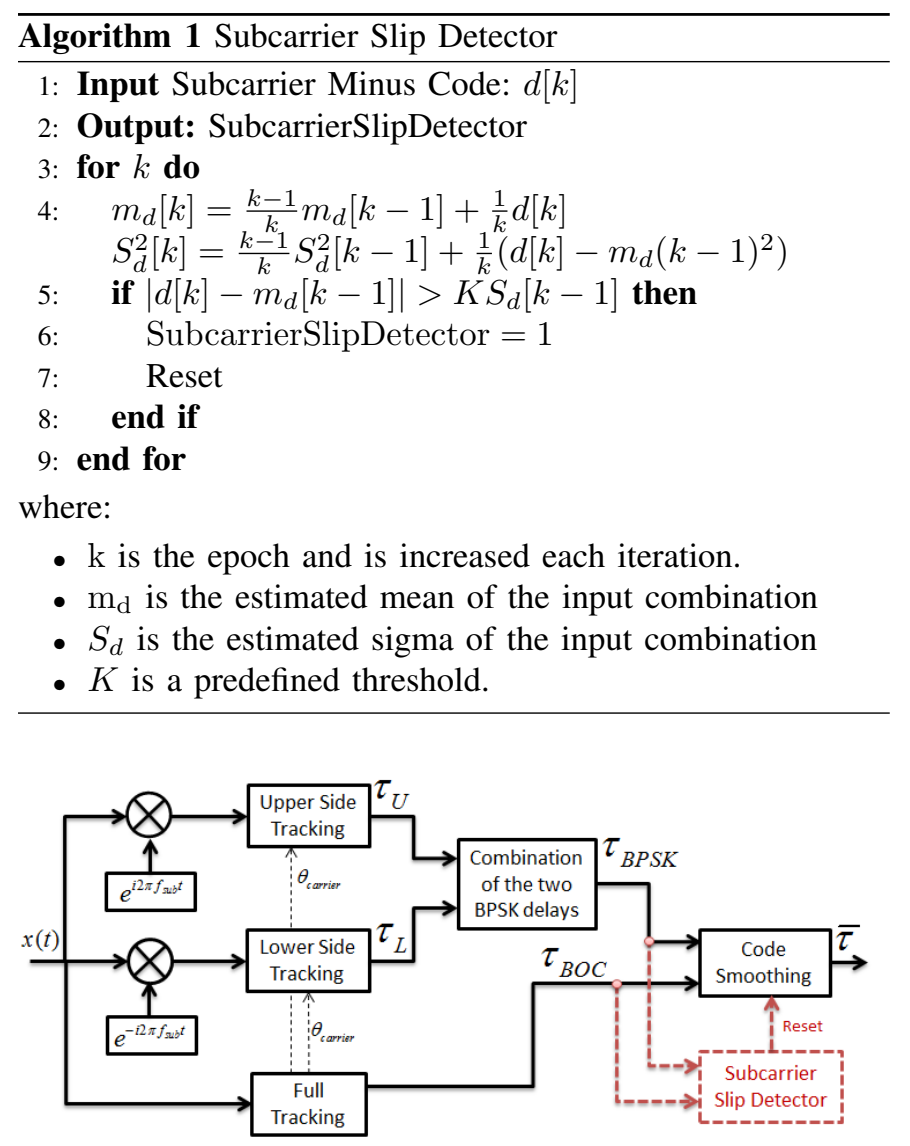

Fig. 3. Code-Subcarrier Smoothing with Subcarrier Slip detection structure

\section{REsults}

In this section the code smoothing results are presented. The common receiver parameters are:

- $\operatorname{BOCcos}(15,2.5)$

- CodePeriod $=0.01$ seconds

- $f s=65 \mathrm{Msps}$

- $\mathrm{BWPLL}=10 \mathrm{~Hz}$

- $\mathrm{BWDLL}_{\mathrm{BPSK}}=1 \mathrm{~Hz}$

- $\mathrm{BWDLL}_{\mathrm{BOC}}=1 \mathrm{~Hz}$

- EarlyLateSpacingBPSK = 0.2 chips

- EarlyLateSpacingBOC $=0.041$ chips

- $\mathrm{K}=4$ (Subcarrier Slip Threshold)

The technique proposed in this paper is compared to Bump Jump algorithm (BJ), Double Estimator technique (DET), the typical Full BOC (Full) Early minus Late and the Dual BPSK (BPSK) and also the smoothing without the Subcarrier detector. When the algorithm detects a false lock, it is indicated using a asterisk marker. All the techniques have been implemented using the same configuration: Early late spacing, DLL bandwidth and discriminators.

Two different scenarios have been simulated in Matlab. The first scenario is based on the land mobile DLR channel [14]. Particularly a suburban vehicular scenario has been simulated. Fig. 4 shows the relative power of the line-of-sight. The same scenario has been tested for different values of $\mathrm{C} / \mathrm{No}$.

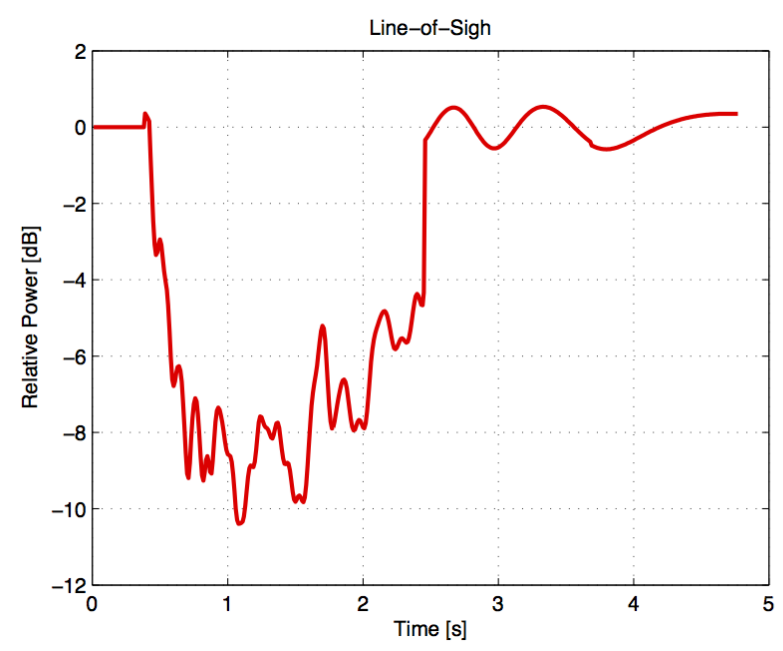

Fig. 4. DLR channel: Line-of-Sigh

The second scenario, or False Lock scenario is shown in Fig. 5. It is a particular channel model composed by the LOS and one multipath ray in order to cause a subcarrier slip. After 1.62 seconds a multipath appears with zero delay and at the same time instant the LOS disappears. The multipath move the tracking point 10 meters in 5 seconds. Then, the multipath disappears and the LOS comes back. The subcarrier measurements remains in a different peak and the code measurements goes back to the main peak. Therefore, a subcarrier slip takes place.
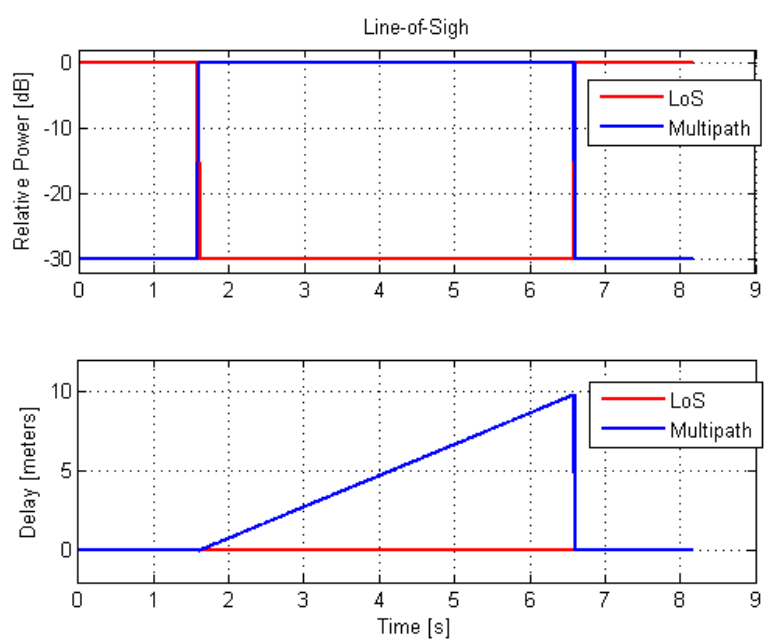

Fig. 5. False Lock channel

Fig. 6 shows the tracking error of the False Lock channel for different values of $C / N o$. At the bottom a high $C / N o$ has been simulated. As it can be seen, when the multipath disappears and the LOS appears, all the techniques go to the main peak solving the ambiguity, except the Full BOC as it is totally ambiguous. It should be remarked that the Subcarrier Slip detector declares a subcarrier-slip when the LOS appears. 
At the middle, $C / N o 30 \mathrm{~dB}-\mathrm{Hz}$, the Bump Jumping and the DET present some undesired jumps due to the noise and the punctual difference between the DLL and the SLL. The proposed Smoothed technique with the subcarrier slip detector does not suffer any jump. However the convergence time after the subcarrier-slip is slightly higher.

When a really low $C / N o$ is simulated (at the top), one may see that the Bump Jumping suffers several jumps as well as the DET. The proposed detector is declaring four false subcarrier slips (instead of one) due to the huge biases introduced by the BPSK measurements. Nevertheless it presents a good behaviour.

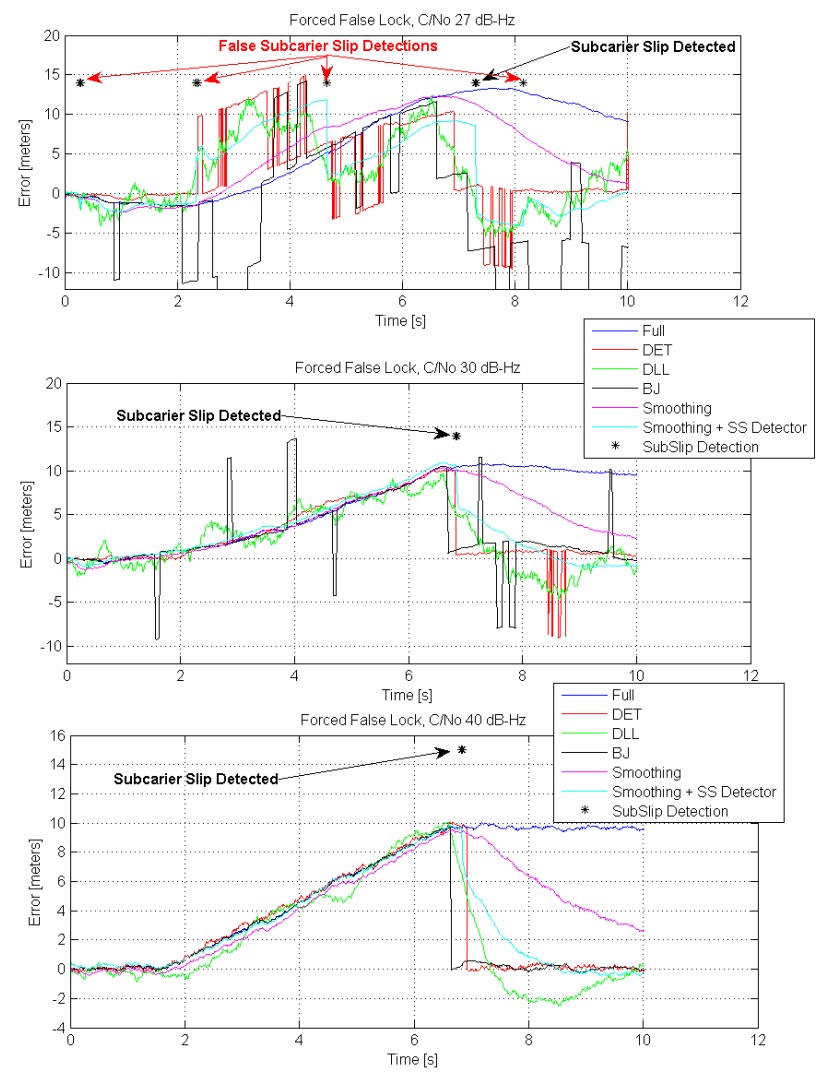

Fig. 6. Time error in False Lock channel

Fig. 7 shows the time error of the DLR channel model for different values of $C / N o$. It should be noted that the subcarrier slip detector has not detected any subcarrier slip. This is fully expected as the Full BOC does not suffer from any false lock. However, both the Bump Jumping and the DET presents some jumps caused by the multipath. The BPSK measurements are much more affected than the Full ones. The channel does not causes any False lock.

The possibility of false lock during the tracking is really unlikely but it has to be taken into account. Therefore, this paper presents a new Subcarrier Slip detector that is able to reset the smoothing when the tracking point goes from one peak to another.
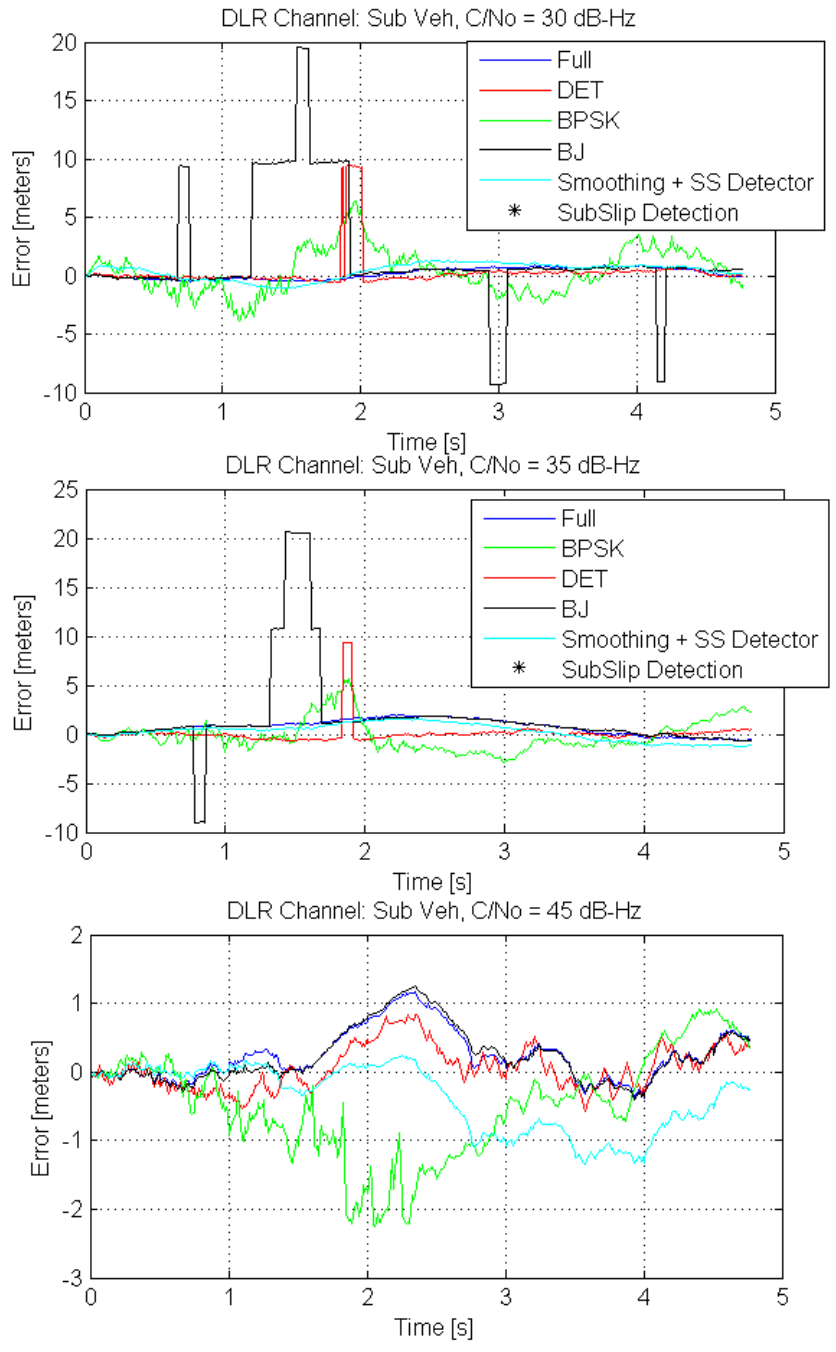

Fig. 7. Time error in DLR channel

\section{CONCLUSIONS}

The work carried out in this paper can be seen as an improvement code-subcarrier technique presented in [8]. It takes advantage of the possibility of independent code and subcarrier tracking of BOC signals. The new tracking method is based on the smoothing strategy: the code measurements, achieved tracking the BOC signal as two BPSK, are smoothed with the subcarrier or Full BOC measurements.

If there is a constant bias between the unambiguous and the ambiguous measurements, the smoothed strategy follows the mean value of the unambiguous one. For instance, when the signal passes through a filter with non-continuous group delay. Since the bias is the same for all the satellites it does not affect the PVT solution.

Several simulations, using the DLR channel model and specific channel demonstrate the good behaviour of the codesubcarrier smoothing with the subcarrier slip detector, achieving a similar behaviour as the Full BOC but mitigating the ambiguity. Besides, the proposed technique is insensitive to 
possible biases between the subcarrier and the code caused by the Rx filtering or even the Tx filters.

After the results presented in this paper, one of the future lines of research is the improvement of the code loop. One of the advantages of the structure presented in [8] is the capability of different code loops strategies. It is much more affected by the multipath effect than the subcarrier loop. Therefore, one may think on the implementation of strobe correlators in order to reduce the multipath effect. Since the code measurements are smoothed with the subcarrier ones, the penalty in the tracking jitter, introduced by the strobe correlators, could be compensated.

\section{ACKNOWLEDGEMENTS}

This work was supported in part by the Spanish Ministry of Economy and Competitiveness projects TEC 2011-28219 and EIC-ESA-2011-0080.

\section{REFERENCES}

[1] P. Fine and W. Wilson, "Tracking algorithm for GPS offset carrier signals," in Proc. of the 1999 National Technical Meeting of The Institute of Navigation, 1999.

[2] N. Martin, V. Leblond, G. Guillotel, and V. Heiries, "BOC (x, y) signal acquisition techniques and performances," in Proc. of ION GPS/GNSS, 2003, pp. 188-198.

[3] P. M. Fishman and J. W. Betz, "Predicting performance of direct acquisition for the M-code signal," in Proc. of ION NTM, 2000, pp. 574-582.

[4] A. Burian, E. Lohan, and M. Renfors, "BPSK-like methods for hybridsearch acquisition of galileo signals," IEEE International Conference on Communications ICC '06, vol. 11, pp. 5211-5216, 2006.

[5] V. Heiries, D. Roviras, L. Ries, and V. Calmettes, "Analysis of non ambiguous BOC signal acquisition performance Acquisition," Proc. of ION ITM, pp. 2611 - 2622, 2004.

[6] M. Hodgart, P. Blunt, and M. Unwin, "The optimal dual estimate solution for robust tracking of Binary Offset Carrier (BOC) modulation," in Proc. of ION GNSS, 2007, pp. 1017-1027.

[7] C. Palestini, "Synchronization and detection techniques for navigation and communication systems," Ph.D. dissertation, Univ. of Bologna, 2010.

[8] M. Navarro-Gallardo, G. Seco-Granados, G. López-Risueño, and M. Crisci, "Code Smoothing for BOC Ambiguity Mitigation," Proc. AIAA International Communication Satellite Systems Conference (ICSSC), 2013.

[9] C. Gunther and P. Henkel, "Reduced-noise ionosphere-free carrier smoothed code," IEEE Transactions on Aerospace and Electronic Systems, vol. 46, no. 1, pp. 323-334, 2010.

[10] S. Sen and J. Rife, "Nonlinear filter for ionosphere divergence error reduction in LAAS," IEEE Transactions on Aerospace and Electronic Systems, vol. 48, no. 2, pp. 981-990, 2012.

[11] E. D. Kaplan and C. Hegarty, Understanding GPS: principles and applications. Artech House Publishers, 2005.

[12] G. Blewitt, "An Automatic Editing Algorithm for GPS Data," Geophysical Research Letters, vol. 17, no. 3, pp. 199-202, 1990.

[13] J. Sanz-Subirana, J. Juan-Zornoza, and M. Hernández-Pajares, GNSS Data Processing. ESA Communications, 2013.

[14] ITU-R, Propagation data required for the design of Earth-space land mobile telecommunication systems, $\mathrm{P}$. Series, Ed. Radiowave propagation, 2009. 